\title{
Comparative study of imaging and pathological evaluation of pneumonic mucinous adenocarcinoma
}

\author{
JUN HAN $^{1 *}$, CHONGCHONG WU ${ }^{2 *}$, YUXIN WU ${ }^{3 *}$, HUI DENG $^{4}$, \\ $\mathrm{JIE} \mathrm{GAO}^{5}$, HUA HAN ${ }^{6}$ and XINYING XUE \\ ${ }^{1}$ Department of Radiology, The Third Affiliated Hospital of Chongqing Medical University, Chongqing 401120; \\ ${ }^{2}$ Department of Radiology, Chinese PLA General Hospital, Beijing 100853; ${ }^{3}$ Department of \\ Radiology, The Traditional Chinese Medicine Hospital of Changshou District, Chongqing 401220; \\ ${ }^{4}$ Department of Respiratory Diseases, Beijing Shijitan Hospital, Capital Medical University, Beijing 100038; \\ ${ }^{5}$ Department of Pathology, Chinese PLA General Hospital, Beijing 100853; ${ }^{6}$ Department of Radiology, \\ The Third Affiliated Hospital of Jinzhou Medical University, Jinzhou, Liaoning 121001, P.R. China
}

Received July 17, 2019; Accepted November 12, 2020

DOI: $10.3892 / \mathrm{ol} .2020 .12386$

\begin{abstract}
Patients with pneumonia-type lung cancer (PTLC) do not exhibit specific clinical features, which makes imaging diagnosis difficult. Therefore, the aetiology of the pathological changes occurring during PTLC remains unclear. The current study aimed to explore the possible mechanism of PTLC formation by CT scans and pathological analysis of the lungs. A retrospective analysis was conducted on the CT and pathological data of 17 cases of PTLC. The diagnosis of lung cancer was confirmed by pathology. The CT scans of nine patients indicated diffuse distribution of lesions in the lungs, whereas those of three patients indicated single-lung multi-leaf distribution, and those of the remaining five patients included single-leaf distribution. All patients demonstrated increased plaque or patchy density in the majority of the lesions located near the heart. The pathological types of the identified tumours were mucinous adenocarcinoma with adherent growth as the main sub-type. A large number of mucus lakes were observed, containing floating tumour cells, as determined by optical microscopy. In addition, a number of tumour cells were located in the residual alveolar wall of the observed mucus lakes.
\end{abstract}

Correspondence to: Professor Hua Han, Department of Radiology, The Third Affiliated Hospital of Jinzhou Medical University, 2 Heping Road Section 5, Jinzhou, Liaoning 121001, P.R. China

E-mail: jzhhh@163.com

Professor Xinying Xue, Department of Respiratory Diseases, Beijing Shijitan Hospital, Capital Medical University, 10 Tieyi Road, Yangfangdian, Haidian, Beijing 100038, P.R. China

E-mail: xuexinying2988@bjsjth.cn

${ }^{*}$ Contributed equally

Key words: lung computed tomography, mucinous adenocarcinoma, pathology, pneumonic mucinous adenocarcinoma
The results of the present study suggested that the mucinous adenocarcinoma tumour cells produced substantial quantities of mucus, and that the cells were scattered and planted along with the mucus through the airway, which led to possible development of pneumonia-type mucinous adenocarcinoma.

\section{Introduction}

Lung cancer is one of the most common tumour types and remains the leading cause of cancer-related death worldwide, with a 5-year survival rate of only $16 \%$ in 2013 (1). The occurrence of lung cancer is closely associated with smoking (2). The incidence of lung adenocarcinoma is the highest among all subtypes of lung cancer and has surpassed that of squamous cell carcinoma (3), and the pathology of PTLC often indicates lung adenocarcinoma. Pneumonia-type lung cancer (PTLC) is often misdiagnosed as inflammation and its treatment is usually delayed (4). Therefore, accurate identification and rapid diagnosis of this disease is essential to improve patient survival.

Several mechanisms of lung cancer metastasis have been proposed, including hematogenous spread, lymphatic metastasis and direct infiltration (5). However, accumulating studies have demonstrated that lung cancer can spread through the trachea (6-9). Gaikwad et al (10) have suggested that aerogenous spread of lung cancer occurs and serves an important role in the staging and management of this disease. At present, the possible mechanism of lung cancer metastasis is considered to be mediated through the airway (10). This facilitates tumour cell adherent growth and metastasis to the distal parts of the trachea, which are located away from the primary site of the malignancy (10).

The current study aimed to investigate the possible mechanism of the formation of PTLC by studying the CT and pathological characteristics of pneumonia-type mucinous adenocarcinoma in order to deepen the understanding of this disease, reduce the misdiagnosis rate and the detection time, and improve the survival rate of the patients. 


\section{Materials and methods}

Patients. A total of 17 patients diagnosed with PTLC between June 2012 and June 2017 were selected from three tertiary hospitals in Beijing (The General Hospital of the People's Liberation Army, The Affiliated Beijing Shijitan Hospital of Capital Medical University and The Peking Union Medical College Hospital; Beijing, China) and their clinical, imaging and pathological data were collected. The present study was approved by the Research Ethics Committee of Beijing Shijitan Hospital affiliated to Capital Medical University [Beijing, China; approval no. sjtkyll-lx-2018(30)]. Since the study was carried out retrospectively, the Ethics Committees of the three hospitals decided to waive the patients' informed consent.

Immunohistochemical staining. The paraffin sections of the lesions were sectioned into $\sim 4-\mu \mathrm{m}$ slices, which were placed on slides overnight at room temperature or dried at $60^{\circ} \mathrm{C}$ for $1 \mathrm{~h}$. The primary anti-thyroid transcription factor-1 (TTF-1) antibody (dilution, 1:50; cat. no. 12373s; Roche Diagnostics) was incubated at $37^{\circ} \mathrm{C}$ for $16 \mathrm{~min}$. The primary antibody and DAB staining solution (Roche Diagnostics) were added to a reagent tray and placed into the Ventana Benchmark XT (Roche Diagnostics) fully automatic immunohistochemical instrument, and the slides were placed directly inside the instrument. Following dyeing, the slides were removed, cleaned with a mild detergent to remove the solution on the cover glass and washed thoroughly with distilled water to remove the residual detergent. After dehydration and cleaning, the cover glass was sealed with sealing agent. The sections were observed under a light microscope with $\times 400$ magnification.

$H \& E$ staining. The sections were dewaxed in xylene for 5-10 min and transferred into the mixture of xylene and pure ethanol (1:1) for $\sim 5 \mathrm{~min}$. Subsequently, the sections were rehydrated in a decreasing series of ethanol $(100,95,85$ and $70 \%$ for 2-5 min each) and transferred to a dye solution through distilled water for staining with haematoxylin for 5-15 min. Eosin dye solution (0.1-0.5\%) was added for 1-5 min, and then the sections were dehydrated by $70,85,95$ and $100 \%$ ethanol for 2-3 min. Finally, the sections were sealed with neutral gum under cover slips. The sections were observed under a light microscope with x100 and x400 magnification.

Imaging and diagnosis. With the exception of three patients who underwent lobectomy, the remaining 14 patients underwent needle biopsies. A total of seven patients underwent rapid on-the-spot evaluation (ROSE) under bronchoscopy. All patients underwent 128 -slice CT with 5-mm thickness, $1.5 \mathrm{~mm}$ scan and parallel coronal and sagittal reconstruction. The diagnosis was made by two deputy chief physicians at the Department of Radiology and was based on the patient clinical symptoms and previous imaging data.

\section{Results}

Clinical characteristics of the patients. Among the 17 recruited patients, 12 were male and five were female. Their age ranged between 35 and 75 years, with a mean age of 60.18 years (Table I). The Tumor-Node-Metastasis stage of all patients was
T4N0M0 (11). The sample included 9 smokers (Table I). The clinical manifestations were cough in 16 patients and absence of apparent symptoms in one patient.

CT scans. A total of nine cases presented with diffuse lung disease, of which one was a case of left upper lobe, two of left lower lobe, two of right upper lobe, two of right lower lobe and one of right middle and lower lobe disease. All patients exhibited multiple patchy, flaky densities and peripheral ground glass density, specifically in the near end of the heart shadow with visible ground glass opacity (14 cases, $82 \%$ ), absence of enlarged lymph nodes and randomly distributed nodule shadows (Fig. 1). In certain patients, air bronchograms (12 cases, $71 \%$ ) and vacuoles (10 cases, 59\%) were observed (Fig. 2). According to the imaging data and clinical symptoms, the disease spread of three patients was confined to the left/lower right lobe, and no distant lymph node or hematogenous metastases were noted. Therefore, the patients were examined with ultrasound/CT guided lung biopsy.

Pathological results. Following surgery, a large amount of mucus was noted by microscopical evaluation, and the tumour cells were scattered in the residual alveolar wall (Fig. 3). Subsequently, the sections were analysed by immunohistochemistry, and the results demonstrated that tumour cells secreting mucus were TTF-1 positive (Fig. 4). The pathological evaluation indicated mucinous adenocarcinoma, and the adherent growth was the dominant type of cancer among all patients. Bronchoscopy was performed in seven cases; a large amount of white foam-like sputum appeared from the left and right main bronchus in the form of a spring. A ROSE was also performed, and the results demonstrated that the full field of view was a tumour (Fig. 5). The cells were examined by multiple biopsies and H\&E staining to confirm that these structures were tumour cells of mucinous adenocarcinoma origin. Tall-cup-like tumour cells that grew in the alveolar wall, secreting a large amount of mucus and leading to the filling of the alveolar cavity, were identified by microscopic examination. Following coughing of the patient and scouring of the mucus, the tumour cells were detached from the wall, and the mucus was spread gradually from the small airway to other airways, lung segments/leaves or to the contralateral lung tissue. When the mucus reached the normal lung tissue, a slightly higher density of ground glass opacity was formed. The floating tumour cells were planted, and a denser patchy solid shadow was observed.

Imaging evaluation. In patient no. 3 (Fig. 6), the CT scan indicated consolidation of the right lower lobe with an unclear edge and a drenched glass opacity around it. The ablated bronchus indicated a dry branch-like change with blocked distal bronchi and slight thickening alterations in the proximal bronchus wall. An additional small airbag cavity was visible, and the inner wall of the cavity was smooth. The CT scan of patient no. 8 (Fig. 7) was indicative of a partial solid tumour in the upper lobe of the right lung. Multiple grinds were noted in the remaining lungs, and a mild uneven enhancement was observed in the right upper lung lesion. Therefore, the CT scans of the two patients suggested that the tumours may be malignant. Multiple biopsies in patient 


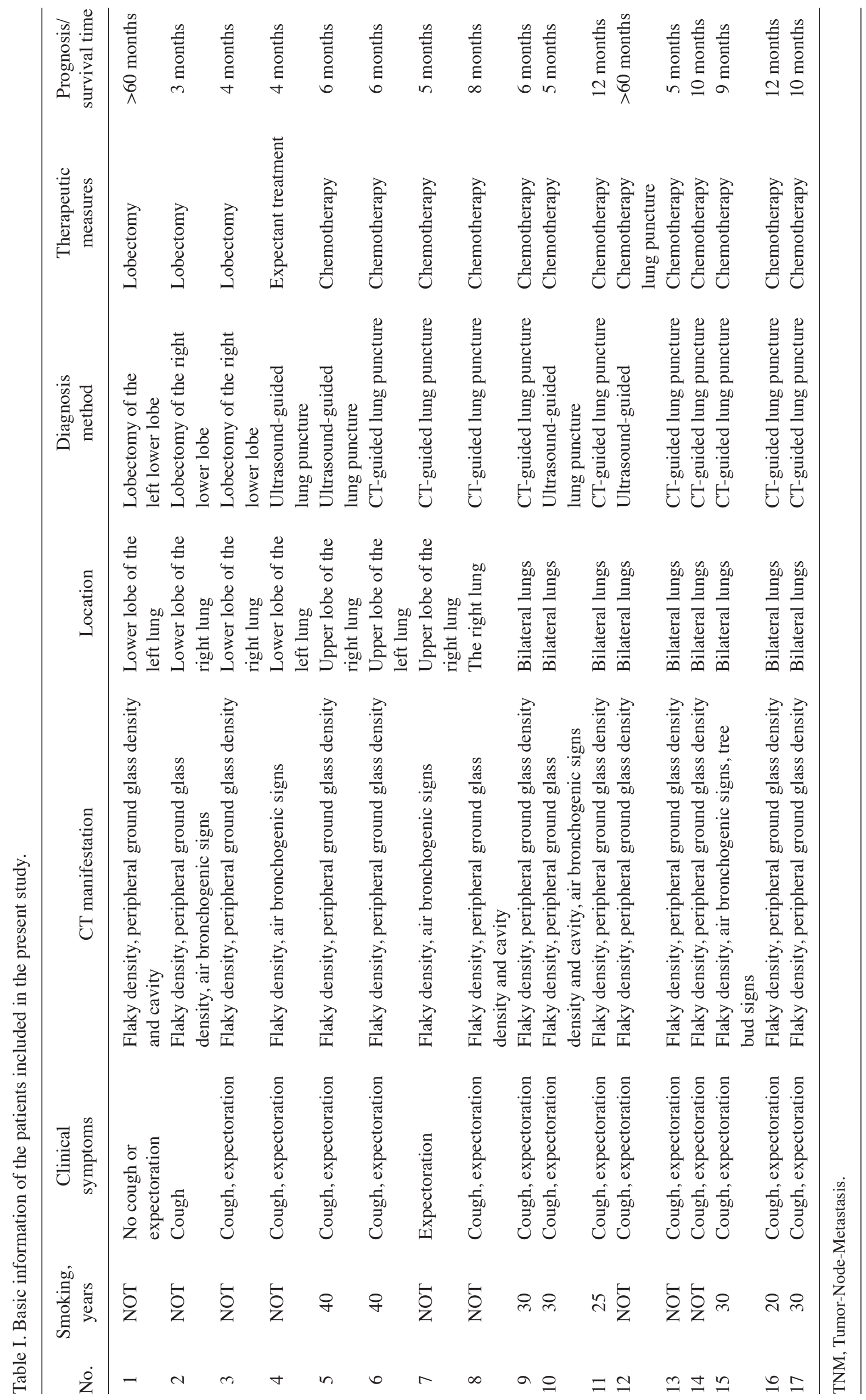




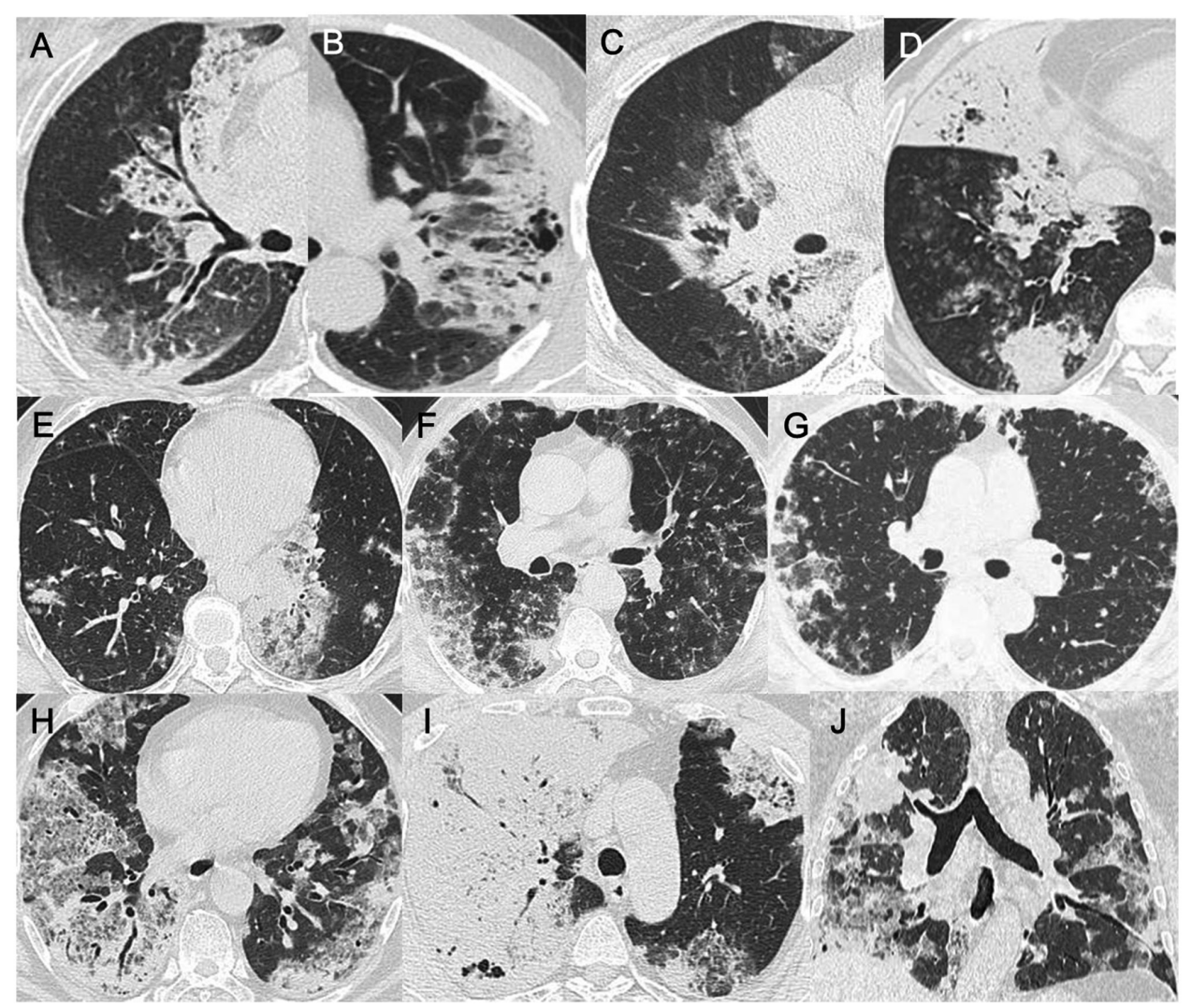

Figure 1. CT manifestations of mucinous adenocarcinoma (A) The distribution of patchy density of mucinous adenocarcinoma in the upper lobe of right lung. (B) The distribution of patchy density of mucinous adenocarcinoma in the upper lobe of the left lung. (C) The distribution of patchy density of mucinous adenocarcinoma in the middle and lower lobe of right lung. (D) The distribution of patchy density of mucinous adenocarcinoma in the middle and lower lobe of right lung. (E) The distribution of patchy density of mucinous adenocarcinoma in the lower lobe of both lungs. (F) The distribution of patchy density of mucinous adenocarcinoma in both lungs. (G) The distribution of patchy density of mucinous adenocarcinoma in both lungs, mainly in the outer zone. (H) Ground glass opacity distribution of mucinous adenocarcinoma of both lungs. (I) Patchy and ground glass opacity distribution of mucinous adenocarcinoma of both lungs. (J) Coronal reconstruction of a bilateral distribution of patchy density in mucinous adenocarcinoma.

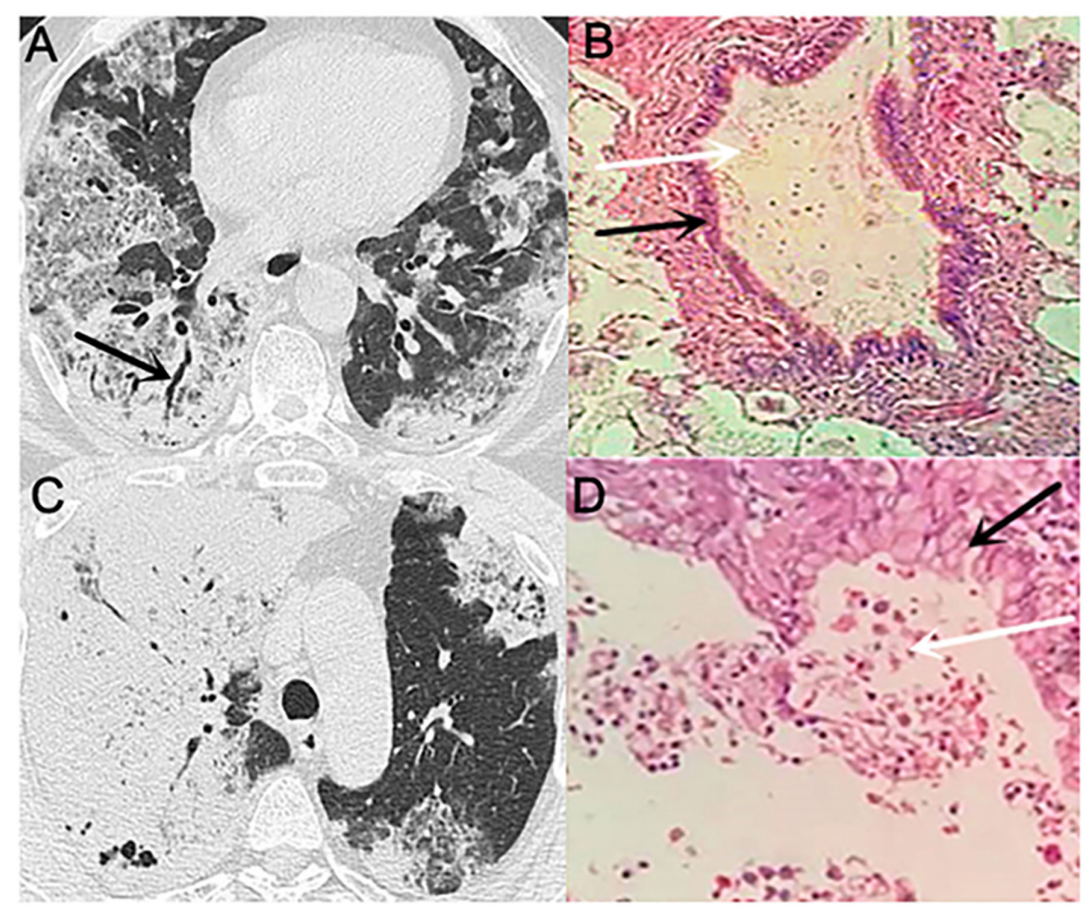

Figure 2. Comparison of CT findings and pathology (A) Black arrow indicates the air bronchogram. (B) H\&E staining of lung mucinous adenocarcinoma tissue obtained by puncture biopsy. Magnification, x100. White arrow indicates the mucus, and the black arrow indicates the wall of the bronchus. (C) Black arrow indicates the vacuole sign. (D) $\mathrm{H} \& \mathrm{E}$ staining of lung mucinous adenocarcinoma tissue. Magnification, $\mathrm{x} 400$. White arrow indicates the mucus and cell nucleus fragments, and black arrow indicates tumour cells. 


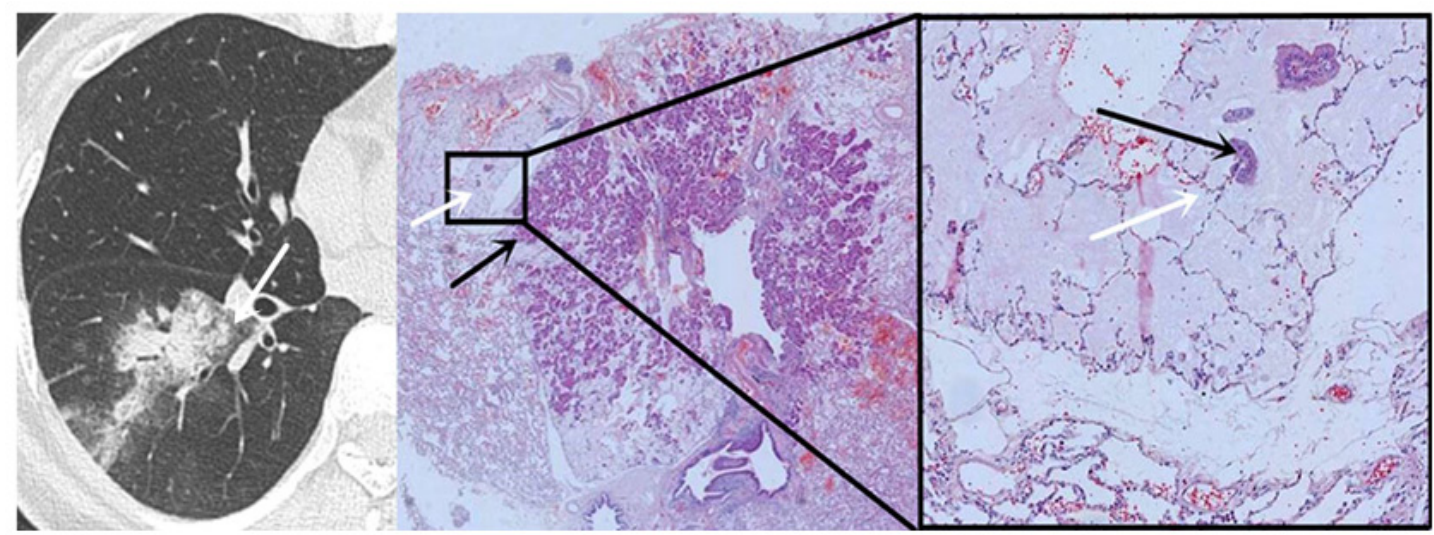

Figure 3. Comparison of ground glass opacity near the heart end with pathology (A) White arrow indicates the ground glass density in the near end of the heart shadow. (B and C) H\&E staining of mucinous adenocarcinoma of the lung. Magnification, (B) x100 and (C) x400. Back arrow indicates the tumour cells, and white arrow indicates the mucus.

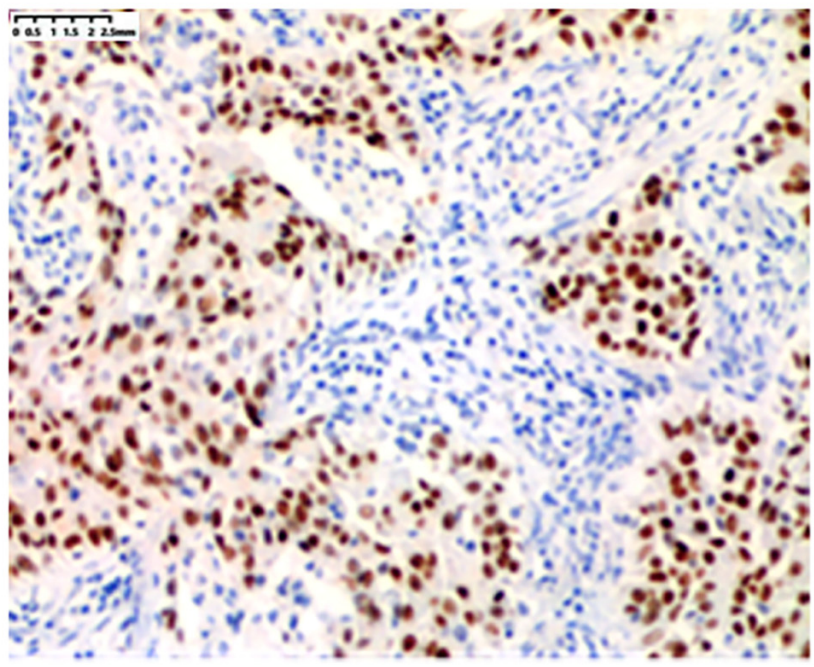

Figure 4. TTF-1 staining. Immunohistochemistry of TTF-1 in mucinous adenocarcinoma tumour cells. The cells were TTF-1 positive. Magnification, x400. TTF-1, thyroid transcription factor-1.

no. 3 and postoperative examination in patient no. 8 indicated mucinous adenocarcinoma. In patient no. 8, a large amount of mucus was noted following microscopical investigation, corresponding to the unenhanced area on the CT. Part of this structure was located in the consolidation zone, where the tumour cells aggregated. The two patients also underwent MRI of the brain and abdomen, which indicated the absence of metastatic lesions.

Subsequently, a 3-month follow-up was conducted. In patient no. 3 , the lesion progressed rapidly within 3 months, and multiple plaques and ground glass opacity were noted in the contralateral lung. The lesions in the left lower lobe of the lung were gradually enlarged, whereas those in the right lower lobe did not change significantly (Fig. 6). It was subsequently revealed that the patient had been sleeping on the left side since the onset of the disease. Patient no. 8 received a right upper lung resection after one week of ineffective anti-inflammatory treatment, and the CT scan 3 months post-surgery revealed that the shadow had mostly disappeared (Fig. 7).
Follow-up results. Among the patients, one received symptomatic treatment, three underwent lobectomy, and the remaining 13 received chemotherapy, which included platinum combined with pemetrexed. Subsequently, the patients were followed up for 5 years (Table I). Of the 17 patients, two were alive at the end of the follow-up period. The longest life span among the patients who succumbed to disease was 12 months.

\section{Discussion}

PTLC is a definition proposed for a specific type of lung cancer that has imaging features comparable to inflammation (12). PTLC represents the morphological process of tumour formation; the tumour gradually develops from single lobe to multiple lobes (13-15). According to a study by Duruisseaux et al (4), PTLC comprises two main histopathological types, namely invasive mucinous adenocarcinoma $(40.0 \%)$ and invasive adenocarcinoma with adherent growth (31.6\%). However, these classifications do not explain the possible mechanism of PTLC formation. Gaikwad et al (10) reviewed the aerogenous metastasis of primary lung adenocarcinoma and suggested that aerogenous spread of tumor cells may exist and that it may be underrecognized. The results of the present study were consistent with the aforementioned study and demonstrated the presence of aerogenous metastasis as multiple flaky and patchy dense structures were evident in the CT scans. The surrounding ring was saturated with ground glass attenuation, especially at the proximal end. The formation of this sign may be associated with the production of mucus by tumour cells and their spread through the airway. Therefore, multiple spots were noted on the CT images.

Lung cancer commonly presents as nodules or masses in CT scans; other malignant signs, such as lobes, burrs and cavities may be visible (16-21). By contrast, PTLC was observed in the present study to be mostly flaky or patchy on CT scans and did not present as a mass or nodule. The near-central ground glass opacity, the air bronchus sign and the vacuole sign are rarely noted in cancerous lymphangitis, and hilar, mediastinal lymph nodes and random distribution of nodules are observed (4). In the present study, the near-central ground glass opacity was the most frequent observation in the 17 patients. A possible explanation for this may be that the tumour cells were 

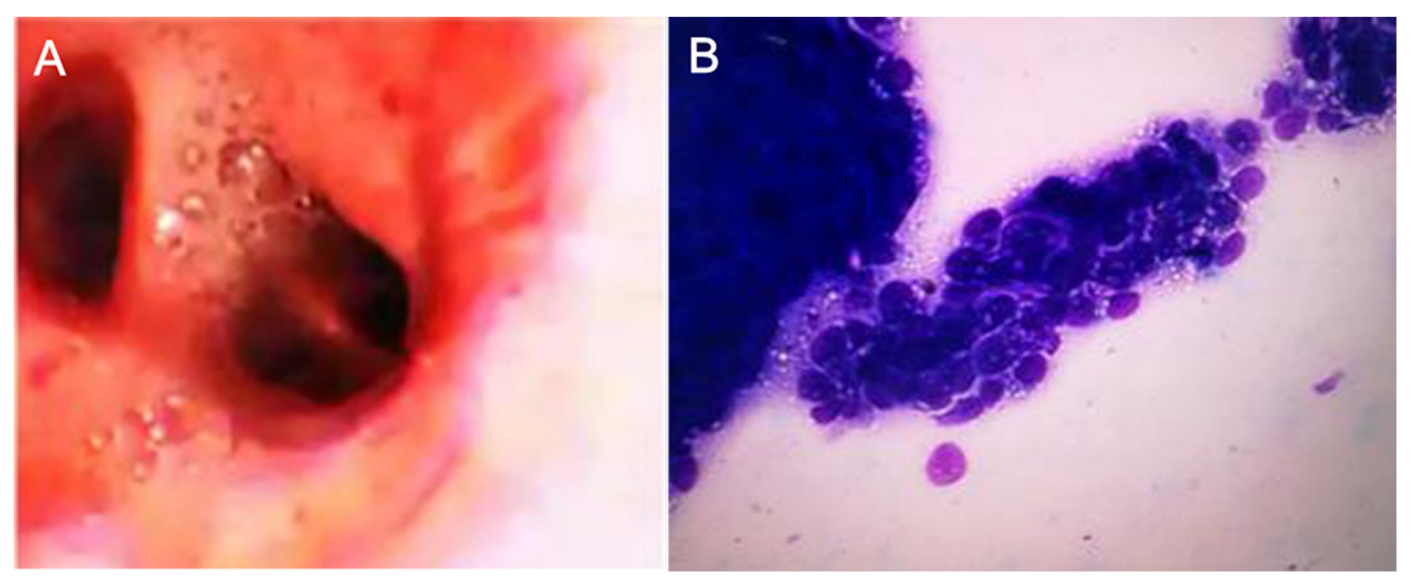

Figure 5. Bronchoscopy and ROSE results. (A) Bronchoscopic examination revealed a fountain of mucus from the trachea. (B) ROSE revealed that the dark stained cells were mucinous adenocarcinoma cancer cells. ROSE, rapid on-the-spot evaluation.

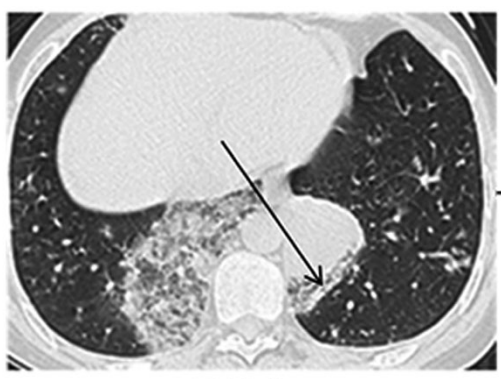

2016-2

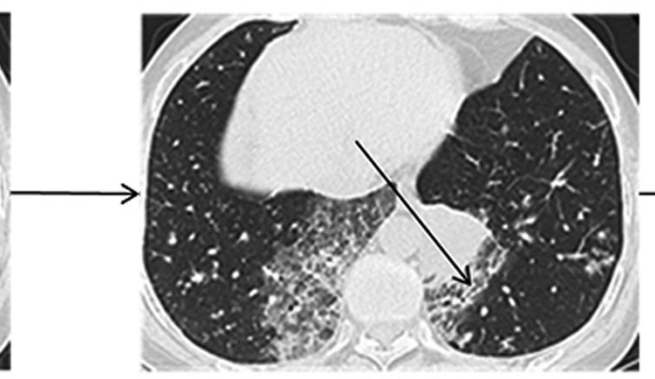

2016-4
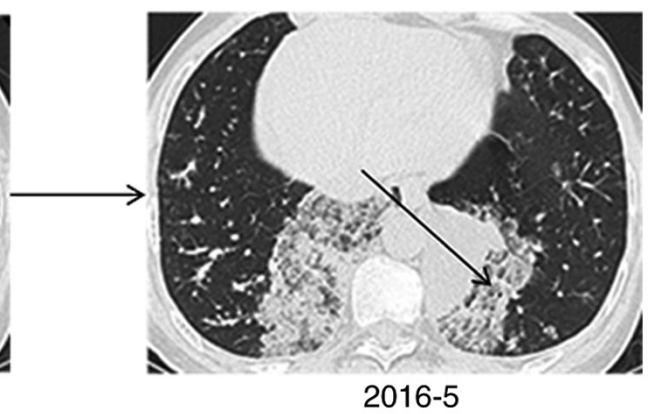

2016-5

Figure 6. Follow-up characteristics of patient no. 3. The lesions in the left lower lobe of the lung were gradually enlarged, whereas those in the right lower lobe did not notably change.

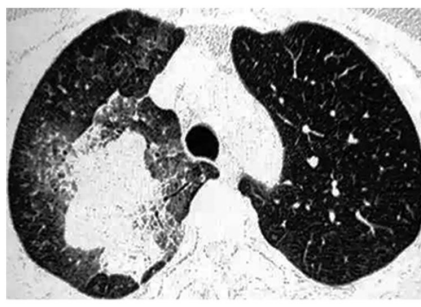

2016-3

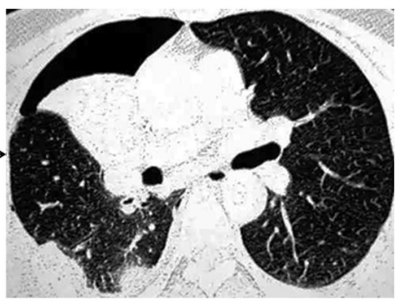

2016-6

Figure 7. Follow-up characteristics of patient no. 8. At 3 months post-lobectomy, the ground glass density disappeared.

dispersed with a large amount of mucus through the airway. However, obstructive pneumonia was difficult to assess since it usually occurs due to the obstruction of the airway, leading to distant frosting or solid shadows. These events were microscopically accompanied by mucus filling and obstruction of the distal small airway. Tumour cells colonized other lung tissues and destroyed the original alveolar space. When the mucus was expelled from the alveoli by coughing or breathing, a translucent and vesicular area was observed. The high incidence of these signs also provided a reliable diagnostic value for the diagnosis of PTLC. Among the patients included in the present study, chemotherapy (platinum plus pemetrexed) was the main treatment for those who preferred non-surgical treatment, although the long-term survival rate of the patients did not appear to improve. Previous studies have demonstrated that certain drugs exert cytotoxic side effects on lung cancer cells and can be used to treat lung cancer. For example, Sani et al (22) have demonstrated that $\mathrm{EO}, \mathrm{CH}_{2} \mathrm{Cl}_{2}$ and hexane components exhibit inhibitory effects on Calu- 6 and Mehr- 80 cells. Luteolin is the main compound extracted from ancient shoucao, a plant used in traditional Chinese medicine, that exhibits considerable cytotoxicity in Calu- 6 and Mehr-80 lung cancer cell lines. Daphedar and Taranath (23) have suggested that high concentrations of silver nanoparticles inhibit the progression of mitotic cells and increase the potential of cell death caused by chromosomal aberrations. This application may provide novel insights into the potential treatment of patients with PTLC.

Chest CT scans have been widely used in the clinical diagnosis of lung cancer $(24,25)$. In addition, the use of CT to 
diagnose PTLC has also been reported (2-4,11,26-30), and a limited number of studies have detailed the possible mechanism of PTLC formation. An important observation of the present study is that it is impossible to perform a biopsy on all plaque shadows or ground glass opacities. However, following multiple means of examination (tracheoscopy, ROSE and pathological examination), the results indicated that the formation of these signs may be associated with the spread of tumour cells through the airway or alveolus. Future studies should combine multiple examination methods to confirm a possible mechanism of action of PTLC. However, a notable limitation of the present study was the low number of cases recruited. In future studies, additional cases should be recruited to verify the main mechanism of action underlying PTLC. The secretion of mucus from the tumour cells requires further investigation based on molecular imaging combined with immunohistochemical analysis. Additionally, Tasdemir et al (31) have reported that EGFR expression levels are upregulated in the majority of patients with NSCLC, and it is an important target in the treatment of NSCLC. The mutation rate of EGFR may provide valuable information, which will be explored in further research.

In conclusion, the results of the present study suggested that PTLC may be considered a potential differential diagnosis when multiple plaques and ground glass opacities are observed around the consolidation area near the heart. These features may include signs such as air bronchus and vacuole. The corresponding pathological type of PTLC is mainly adherent growth-based mucinous adenocarcinoma.

\section{Acknowledgements}

Not applicable.

\section{Funding}

The present study was supported by the National Natural Science Fund Youth Project (grant no. 81700007), the Research and Innovation Fund of The Ministry of Education (grant no. 2018A03026), the Beijing Natural Science Foundation (grant no. 2019A10), the 'Qingmiao' Plan of the Beijing Municipal Hospital Administration (grant no. 2018QM4), The Outstanding Top Talent Fund (grant no. 2019YXBJ1), Capital Health Development Scientific Research Unit Matching Fund (grant no. 2020-2Z-2086) and the Excellent Talents in Beijing Youth Top Team Fund (grant no. 2019YXBJ2).

\section{Availability of data and materials}

The datasets used during the current study are available from the corresponding author on reasonable request.

\section{Authors' contributions}

JH designed the study, analysed the data and contributed to the writing and editing of the manuscript. YW contributed to the experimental design and data analysis. $\mathrm{HH}$ conceived the study and contributed to the first draft of the manuscript. CW and XX participated in the experimental design, conducted the experiments, analysed the data and contributed to drafting the manuscript and revising it for intellectual content. HD participated in performing the experiments. JG analysed the data. All authors read and approved the final manuscript.

\section{Ethics approval and consent to participate}

The present study was conducted in compliance with the institutional policy regarding the protection of patient confidential information and was approved by the Research Ethics Committee of Beijing Shijitan Hospital affiliated to Capital Medical University [Beijing, China; approval no. sjtkyll-lx-2018(30)]. All procedures were performed in accordance with the approved guidelines of Beijing Shijitan Hospital affiliated to Capital Medical University.

\section{Patient consent for publication}

Not applicable.

\section{Competing interests}

The authors declare that they have no competing interests.

\section{References}

1. Linnerth-Petrik NM, Walsh SR, Bogner PN, Morrison C and Wootton SK: Jaagsiekte sheep retrovirus detected in human lung cancer tissue arrays. BMC Res Notes 7: 160, 2014.

2. Liu J, Shen J, Yang C, He P, Guan Y, Liang W and He J: High incidence of EGFR mutations in pneumonic-type non-small cell lung cancer. Medicine (Baltimore) 94: e540, 2015.

3. Casali C, Rossi G, Marchioni A, Sartori G, Maselli F, Longo L, Tallarico E and Morandi U: A single institution-based retrospective study of surgically treated bronchioloalveolar adenocarcinoma of the lung: Clinicopathologic analysis, molecular features, and possible pitfalls in routine practice. $\mathrm{J}$ Thorac Oncol 5: 830-836, 2010.

4. Duruisseaux M, Antoine M, Rabbe N, Poulot V, Fleury-Feith J, Vieira T, Lavolé A, Cadranel J and Wislez M: The impact of intracytoplasmic mucin in lung adenocarcinoma with pneumonic radiological presentation. Lung Cancer 83: 334-340, 2014.

5. Wislez M, Antoine M, Baudrin L, Poulot V, Neuville A, Pradere M, Longchampt E, Isaac-Sibille S, Lebitasy MP and Cadranel J: Non-mucinous and mucinous subtypes of adenocarcinoma with bronchioloalveolar carcinoma features differ by biomarker expression and in the response to gefitinib. Lung Cancer 68: 185-191, 2010.

6. Pelosi G: The new taxonomy of lung adenocarcinoma stemming from a multidisciplinary integrated approach: Novel pathology concepts and perspectives. J Thorac Oncol 6: 241-243, 2011.

7. Wislez M, Antoine M, Rabbe N, Gounant V, Poulot V, Lavolé A, Fleury-Feith $\mathrm{J}$ and Cadranel J: Neutrophils promote aerogenous spread of lung adenocarcinoma with bronchioloalveolar carcinoma features. Clin Cancer Res 13: 3518-3527, 2007.

8. Seo JB, Im JG, Goo JM, Chung MJ and Kim MY: Atypical pulmonary metastases: Spectrum of radiologic findings. Radiographics 21: 403-417, 2001.

9. Herold CJ, Bankier AA and Fleischmann D: Lung metastases. Eur Radiol 6: 596-606, 1996.

10. Gaikwad A, Souza CA, Inacio JR, Gupta A, Sekhon HS, Seely JM, Dennie C and Gomes MM: Aerogenous metastases: A potential game changer in the diagnosis and management of primary lung adenocarcinoma. AJR Am J Roentgenol 203: W570-W582, 2014.

11. Lababede $\mathrm{O}$ and Meziane MA: The eighth edition of TNM staging of lung cancer: Reference chart and diagrams. Oncologist 23: 844-848, 2018.

12. Wislez M, Massiani MA, Milleron B, Souidi A, Carette MF, Antoine $\mathrm{M}$ and Cadranel J: Clinical characteristics of pneumonic-type adenocarcinoma of the lung. Chest 123: 1868-1877, 2003. 
13. Garfield DH, Cadranel JL, Wislez M, Franklin WA and Hirsch FR: The bronchioloalveolar carcinoma and peripheral adenocarcinoma spectrum of diseases. J Thorac Oncol 1: 344-359, 2006

14. Manson GV and Ma PC: Response to pemetrexed chemotherapy in lung adenocarcinoma-bronchioloalveolar carcinoma insensitive to erlotinib. Clin Lung Cancer 11: 57-60, 2010.

15. Garfield DH, Cadranel J and West HL: Bronchioloalveolar carcinoma: The case for two diseases. Clin Lung Cancer 9: 24-29, 2008.

16. Youlden DR, Cramb SM and Baade PD: The international epidemiology of lung cancer: Geographical distribution and secular trends. J Thorac Oncol 3: 819-831, 2008.

17. Mavi A, Lakhani P, Zhuang H, Gupta NC and Alavi A: Fluorodeoxyglucose-PET in characterizing solitary pulmonary nodules, assessing pleural diseases, and the initial staging, restaging, therapy planning, and monitoring response of lung cancer. Radiol Clin North Am 43: 1-21, ix, 2005.

18. Acker MR and Burrell SC: Utility of 18F-FDG PET in evaluating cancers of lung. J Nucl Med Technol 33: 69-74; quiz 75-7, 2005.

19. Travis WD, Brambilla E, Nicholson AG, Yatabe Y, Austin JHM, Beasley MB, Chirieac LR, Dacic S, Duhig E, Flieder DB, et al: The 2015 world health organization classification of lung tumors: Impact of genetic, clinical and radiologic advances since the 2004 classification. J Thorac Oncol 10: 1243-1260, 2015.

20. Raz DJ, He B, Rosell R and Jablons DM: Bronchioloalveolar carcinoma: A review. Clin Lung Cancer 7: 313-322, 2006.

21. Mornex JF, Thivolet F, De las Heras M and Leroux C: Pathology of human bronchioloalveolar carcinoma and its relationship to the ovine disease. Curr Top Microbiol Immunol 275: 225-248, 2003.

22. Sani TA, Mohammadpour E, Mohammadi A, Memariani T, Yazdi MV, Rezaee R, Calina D, Docea AO, Goumenou M, Etemad L and Shahsavand S: Cytotoxic and apoptogenic properties of dracocephalum kotschyi aerial part different fractions on calu-6 and mehr-80 lung cancer cell lines. Farmacia 65: 189-199, 2017.

23. Daphedar A and Taranath TC: Characterization and cytotoxic effect of biogenic silver nanoparticles on mitotic chromosomes of Drimia polyantha (Blatt. \& McCann) stearn. Toxicol Rep 5: 910-918, 2018
24. Qi Y, Zhang Q, Huang Y and Wang D: Manifestations and pathological features of solitary thin-walled cavity lung cancer observed by CT and PET/CT imaging. Oncol Lett 8: 285-290, 2014.

25. Miyake H, Matsumoto A, Terada A, Yoshida S, Takaki H and Mori H: Mucin-producing tumor of the lung: CT findings. J Thorac Imaging Spring 10: 96-98, 1995.

26. Sato K, Ueda Y, Shikata H and Katsuda S: Bronchioloalveolar carcinoma of mixed mucinous and nonmucinous type: Immunohistochemical studies and mutation analysis of the p53 gene. Pathol Res Pract 202: 751-756, 2006.

27. Isobe K, Hata Y, Iwata M, Ishida F, Kaburaki K, Gocho K, Kobayashi M, Sakaguchi S, Satou D, Sano G, et al: An autopsied case of mucinous bronchioloalveolar carcinoma associated with multiple thin-walled cavities. Nihon Kokyuki Gakkai Zasshi 47: 512-517, 2009 (In Japanese).

28. Woodring JH: Unusual radiographic manifestations of lung cancer. Radiol Clin North Am 28: 599-618, 1990.

29. Osoegawa A, Kometani T, Nosaki K, Ondo K, Hamatake M, Hirai F, Seto T, Sugio K and Ichinose Y: LKB1 mutations frequently detected in mucinous bronchioloalveolar carcinoma. Jpn J Clin Oncol 41: 1132-1137, 2011.

30. Kadota K, Yeh YC, D'Angelo SP, Moreira AL, Kuk D, Sima CS, Riely GJ, Arcila ME, Kris MG, Rusch VW, et al: Associations between mutations and histologic patterns of mucin in lung adenocarcinoma: Invasive mucinous pattern and extracellular mucin are associated with KRAS mutation. Am J Surg Pathol 38: 1118-1127, 2014.

31. Tasdemir S, Taheri S, Akalin H, Kontas O, Onal O and Ozkul Y: Increased EGFR mRNA expression levels in non-small cell lung cancer. Eurasian J Med 51: 177-185, 2019.

This work is licensed under a Creative Commons Attribution-NonCommercial-NoDerivatives 4.0 International (CC BY-NC-ND 4.0) License. 